\title{
BEST CONSTANTS IN ONE-SIDED WEAK-TYPE INEQUALITIES
}

\author{
A. B. J. Kuijlaars
}

\begin{abstract}
We prove that the best constant in the one-sided weak 1-1 inequality for the Hilbert transform $m(\{\tilde{f}(x) \geq \lambda\}) \leq K\|f\|_{1} / \lambda$ is equal to $K=1$. This is used to obtain the best constant in an inequality of P. B. Borwein dealing with logarithmic derivatives of rational functions. In addition, we find best constants in various related inequalities.
\end{abstract}

\section{Introduction}

The starting point of this paper is an inequality of Borwein [2] for the logarithmic derivative of rational functions. He showed that

$$
m\left(\left\{x \in \mathbf{R}: \frac{r_{n}^{\prime}(x)}{r_{n}(x)} \geq \lambda\right\}\right) \leq \frac{8 n}{\lambda}
$$

for all rational functions $r_{n}$ of degree $\leq n$ and $\lambda>0$. See also [3, page 347]. Here $m$ denotes the Lebesgue measure on the real line. In [2], the inequality (1.1) was used to obtain lower bounds for the rate of approximation to $\exp (-x)$ on $[0, \infty)$ by rationals. The exact rate was obtained somewhat later by Gonchar and Rakhmanov [7] using different methods.

The constant 8 in (1.1) is not optimal, and Borwein, Rakhmanov, and Saff [4] conjectured that the best constant should be $2 \pi$, and it is one of the goals of this paper to prove that indeed

$$
m\left(\left\{x \in \mathbf{R}: \frac{r_{n}^{\prime}(x)}{r_{n}(x)} \geq \lambda\right\}\right) \leq \frac{2 \pi n}{\lambda},
$$

and $2 \pi$ cannot be replaced by any smaller constant. We note that from (1.2), we easily get a different proof for one of the results in [4]. See Corollary 6 below.

The approach to prove (1.2) is based on the observation that (1.2) is related to a weak-type inequality for the classical Hilbert transform on the real line $f \mapsto \tilde{f}$ where

$$
\tilde{f}(x):=\frac{1}{\pi} \int_{-\infty}^{\infty} \frac{f(t)}{x-t} d t .
$$

Recall that the Hilbert transform is a bounded linear operator on $L^{p}$ for every $p \in$ $(1, \infty)$, but not on $L^{1}$. Instead, we have a weak $1-1$ inequality: there is a constant $C>0$ such that for every $f \in L^{1}$ and $\lambda>0$,

$$
m(\{x \in \mathbf{R}:|\tilde{f}(x)| \geq \lambda\}) \leq \frac{C}{\lambda}\|f\|_{1} .
$$

Received August 6, 1996, revised November 5, 1997.

1991 Mathematics Subject Classification: Primary 44A15, Secondary 31A05, 41A20.

Key words and phrases: Hilbert transform, weak-type inequality, rational approximation. 
The best constant in (1.3) was determined by Davis [6], who found

$$
C=\pi^{3}\left(\int_{0}^{\pi}|\log \tan \theta / 2| d \theta\right)^{-1}=\frac{1+3^{-2}+5^{-2}+\cdots}{1-3^{-2}+5^{-2}-\cdots} .
$$

Davis used probabilistic methods to prove this result. Later, Baernstein [1] gave an analytic proof. It should be emphasized that (1.4) is the best constant for real-valued $L^{1}$ functions. The best constant for complex-valued $L^{1}$ functions is unknown.

The inequality (1.2) is related to the one-sided weak 1-1 inequality for the Hilbert transform

$$
m(\{x \in \mathbf{R}: \tilde{f}(x) \geq \lambda\}) \leq \frac{K}{\lambda}\|f\|_{1} .
$$

We are going to extend Baernstein's analytic method to prove that the best constant in (1.5) is $K=1$, and this will be the main tool in proving (1.2). More generally, we determine the best constant $K=K(\sigma)$ in (1.5) for the class of functions $f \in L^{1}$ satisfying

$$
\int_{-\infty}^{\infty} f(t) d t=\sigma\|f\|_{1}
$$

where $\sigma \in[-1,1]$. This enables us to obtain the best constants in inequalities like (1.1) for ray sequences of rational functions. This means that for some $\alpha, \beta>0$, we have $r_{n}=p_{n} / q_{n}$ with $\operatorname{deg} p_{n} \leq \alpha n$ and $\operatorname{deg} q_{n} \leq \beta n$.

The sharp constants we find for the Hilbert transform are also sharp in the corresponding inequalities for conjugate functions on the unit circle. In this paper, we are only dealing with the Hilbert transform since this is what is needed for the inequalities concerning logarithmic derivatives of rational functions. The changes that are required for the conjugate functions are left to the reader.

We will formulate our results more precisely in the next section. The main results are Theorem 1 on the Hilbert transform from which various weak-type inequalities follow, and Theorem 5 on the logarithmic derivatives of rational functions. A major role in the proof of Theorem 1 is played by a certain subharmonic function in the complex plane. Its definition and properties are given in Section 3. We remark that a similar, though somewhat simpler, function was used by Baernstein [1] to obtain the best constant in the two-sided inequality. Finally, the proofs of the results are given in Sections 4 and 5.

\section{Statement of results}

2.1. Best constants for the Hilbert transform. In what follows, $L^{1}$ denotes the space of real-valued integrable functions on the real line with norm

$$
\|f\|_{1}:=\int_{-\infty}^{\infty}|f(t)| d t, \quad f \in L^{1} .
$$

We also use

$$
I(f):=\int_{-\infty}^{\infty} f(t) d t, \quad f \in L^{1} .
$$

Our main result is 
Theorem 1. Let $\theta \in(-\pi / 2, \pi / 2)$.

(a) For every $f \in L^{1}$ and $\lambda>0$, we have

$$
m(\{x \in \mathbf{R}: \tilde{f}(x) \geq \lambda\}) \leq \frac{1}{\lambda}\left(\frac{1}{\cos \theta}\|f\|_{1}-\frac{2 \theta}{\pi \cos \theta} I(f)\right) .
$$

(b) Let $f_{\theta}$ be given by

$$
f_{\theta}(x):=\left\{\begin{array}{cl}
\frac{x+\sin \theta}{\sqrt{1-x^{2}}}, & \text { if }-1<x<1, \\
0, & \text { elsewhere. }
\end{array}\right.
$$

Then equality holds in (2.1) for $f=\lambda f_{\theta}$.

Of course, $f_{\theta}$ is not the only function for which equality holds in (2.1). It will follow from the proof of Theorem 1 that more such functions can be found from conformal mappings from the upper half plane $\mathbf{H}$ onto the domain $\Omega_{\theta}$ given by

$$
\Omega_{\theta}:=\{w \in \mathbf{C}: \Im w<1\} \backslash\{i v: v \leq 1-\cos \theta\} .
$$

Let us use $i v+$ to denote the point on the right side of the slit $\{i v: v<1-\cos \theta\}$ and $i v$ - for the point on the left side. Then any conformal map $F$ from $\mathbf{H}$ onto $\Omega_{\theta}$ such that

$$
F(\infty)= \begin{cases}0 & \text { if } \theta=0 \\ 0+ & \text { if } \theta>0 \\ 0- & \text { if } \theta<0\end{cases}
$$

gives rise by means of

$$
f(x):=\Re F(x), \quad-\infty<x<\infty,
$$

to a function $f \in L^{1}$ for which equality holds in (2.1) with $\lambda=1$. The function $f_{\theta}$ from (2.2) corresponds in this sense to the conformal mapping $F$ with additional requirements

$$
F(1)=+\infty+i, \quad F(-1)=-\infty+i .
$$

We state some immediate corollaries of Theorem 1 . Choosing $\theta=0$, we obtain the best constant in the one-sided weak 1-1 inequality (1.5):

Corollary 2. For every $f \in L^{1}$ and $\lambda>0$, we have

$$
m(\{x \in \mathbf{R}: \tilde{f}(x) \geq \lambda\}) \leq \frac{1}{\lambda}\|f\|_{1} .
$$

Equality holds for the function $\lambda f_{0}$.

It is remarkable that in the one-sided inequality (2.5), equality holds for some function $f \not \equiv 0$, since in the two-sided inequality (1.3) with best constant (1.4), equality cannot hold for $f \not \equiv 0$; cf. [6].

For $\theta \rightarrow \pi / 2$, the right-hand side of (2.1) is unbounded unless $I(f)=\|f\|_{1}$, which means that $f$ is non-negative a.e. In that case, the right-hand side tends to $(2 /(\lambda \pi))\|f\|_{1}$, and we obtain a result due to Davis [5].

Corollary 3 (Davis). For every non-negative $f \in L^{1}$ and $\lambda>0$, we have

$$
m(\{x \in \mathbf{R}: \tilde{f}(x) \geq \lambda\}) \leq \frac{2}{\lambda \pi}\|f\|_{1} .
$$


Equality holds for $f=\lambda f_{\pi / 2}$ where

$$
f_{\pi / 2}(x):=\left\{\begin{array}{cl}
\sqrt{\frac{1+x}{1-x}}, & \text { if }-1<x<1 \\
0, & \text { elsewhere. }
\end{array}\right.
$$

and $\lambda=1$.

If we have $I(f)=\sigma\|f\|_{1}$ for some $\sigma \in(-1,1)$, then (2.1) gives

$$
m(\{x \in \mathbf{R}: \tilde{f}(x) \geq \lambda\}) \leq \frac{1}{\lambda}\left(\frac{1}{\cos \theta}-\frac{2 \sigma \theta}{\pi \cos \theta}\right)\|f\|_{1}
$$

for every $\theta \in(-\pi / 2, \pi / 2)$, and we can minimize the constant with respect to $\theta$. A straightforward calculation then gives the following result.

Corollary 4. Let $\sigma \in(-1,1)$. Then there is a unique $\theta=\theta_{\sigma} \in(-\pi / 2, \pi / 2)$ such that

$$
\sin \theta_{\sigma}=\frac{2 \sigma}{\pi}\left(\cos \theta_{\sigma}+\theta_{\sigma} \sin \theta_{\sigma}\right)
$$

Defining

$$
K(\sigma):=\frac{1}{\cos \theta_{\sigma}}-\frac{2 \sigma \theta_{\sigma}}{\pi \cos \theta_{\sigma}}=\frac{2 \sigma}{\pi \sin \theta_{\sigma}}
$$

we have for every $f \in L^{1}$ such that $I(f)=\sigma\|f\|_{1}$ and every $\lambda>0$,

$$
m(\{x \in \mathbf{R}: \tilde{f}(x) \geq \lambda\}) \leq \frac{K(\sigma)}{\lambda}\|f\|_{1} .
$$

The constant $K(\sigma)$ is sharp. In fact, for $f=f_{\theta_{\sigma}}$ as given in (2.2), we have

$$
I\left(f_{\theta_{\sigma}}\right)=\sigma\left\|f_{\theta_{\sigma}}\right\|_{1}
$$

and equality holds in (2.9) with $\lambda=1$ since both sides are equal to 2.

That (2.10) holds can be seen from (2.2) and (2.7). Indeed, by direct calculation

$$
I\left(f_{\theta_{\sigma}}\right)=\int_{-1}^{1} \frac{x+\sin \theta_{\sigma}}{\sqrt{1-x^{2}}} d x=\pi \sin \theta_{\sigma}
$$

and

$$
\left\|f_{\theta_{\sigma}}\right\|_{1}=\int_{-1}^{1}\left|\frac{x+\sin \theta_{\sigma}}{\sqrt{1-x^{2}}}\right| d x=2 \cos \theta_{\sigma}+2 \theta_{\sigma} \sin \theta_{\sigma}
$$

which together with (2.7) gives (2.10).

It is easy to verify that $K(0)=1$ and $K(1)=\lim _{\sigma \rightarrow 1} K(\sigma)=2 / \pi$. It is also easy to see that $K$ is even and decreasing on $[0,1]$. Using these, we find that the Corollaries 2 and 3 are contained in Corollary 4. 
2.2. Best constants for logarithmic derivatives of rational functions. As mentioned in the Introduction, an inequality of the type (1.2) is related to the inequality (2.5). From the more general results in $\S 2.1$, especially Corollary 4 , we obtain sharp inequalities for rational functions $r_{n}=p_{n} / q_{n}$ where $p_{n}$ and $q_{n}$ have more general degrees. We use the function $K$ as defined by (2.7) and (2.8) in Corollary 4.

Theorem 5. Let $\alpha \geq 0, \beta \geq 0, \alpha+\beta>0$, and let $r_{n}=p_{n} / q_{n}$ be a rational function where $\operatorname{deg} p_{n} \leq \alpha n$ and $\operatorname{deg} q_{n} \leq \beta n$. Then for every $\lambda>0$,

$$
m\left(\left\{x \in \mathbf{R}: \frac{r_{n}^{\prime}(x)}{r_{n}(x)} \geq \lambda\right\}\right) \leq \frac{(\alpha+\beta) \pi}{\lambda} K\left(\frac{\alpha-\beta}{\alpha+\beta}\right) n .
$$

Given $\alpha$ and $\beta$, the number $(\alpha+\beta) \pi K((\alpha-\beta) /(\alpha+\beta))$ is asymptotically the best possible, in the sense that for every smaller constant $C$, there is for every large enough $n$, a rational function $r_{n}=p_{n} / q_{n}$ with $\operatorname{deg} p_{n} \leq \alpha n, \operatorname{deg} q_{n} \leq \beta n$ such that for some $\lambda>0$,

$$
m\left(\left\{x \in \mathbf{R}: \frac{r_{n}^{\prime}(x)}{r_{n}(x)} \geq \lambda\right\}\right)>\frac{C n}{\lambda} .
$$

For $\alpha=1, \beta=0$, we use $K(1)=2 / \pi$ to rediscover a result of Borwein [2;3, page $346]$ : for every polynomial $p_{n}$ of degree $\leq n$, we have

$$
m\left(\left\{x \in \mathbf{R}: \frac{p_{n}^{\prime}(x)}{p_{n}(x)} \geq \lambda\right\}\right) \leq \frac{2 n}{\lambda}, \quad \lambda>0 .
$$

That the constant 2 is best possible was shown by Kristiansen [9].

Next, we take $\alpha=\beta=1$ in Theorem 5 . Since $K(0)=1$, it states that for every rational function $r_{n}$ of degree $\leq n$,

$$
m\left(\left\{x \in \mathbf{R}: \frac{r_{n}^{\prime}(x)}{r_{n}(x)} \geq \lambda\right\}\right) \leq \frac{2 \pi n}{\lambda}, \quad \lambda>0,
$$

and that the constant $2 \pi$ is sharp. This proves the conjecture of Borwein, Rakhmanov, and Saff [4].

Finally, we mention that Theorem 5 can be used to give a different proof for Theorem 1.3 in [4]:

Corollary 6 (Borwein-Rakhmanov-Saff). Let $\left\{r_{n}\right\}_{n=1}^{\infty}, r_{n}=p_{n} / q_{n}$ be a sequence of rational functions, such that $\operatorname{deg} p_{n} \leq \alpha n, \operatorname{deg} q_{n} \leq \beta n$. If $R>0$ is such that

$$
\lim _{n \rightarrow \infty}\left\|e^{-n x} r_{n}(x)-1\right\|_{[0, R]}=0
$$

then

$$
R \leq(\alpha+\beta) \pi K\left(\frac{\alpha-\beta}{\alpha+\beta}\right) .
$$

The constant $(\alpha+\beta) \pi K((\alpha-\beta) /(\alpha+\beta))$ is the same as the number $\hat{a}$ used in $[4$, Theorem 1.3]. That this constant is sharp in Corollary 6 was shown very recently by Rakhmanov, Saff, and Simeonov [10]. 


\section{A subharmonic function}

The proof of Theorem 1 depends on the properties of a certain auxiliary function $h=h(w)$ defined in the complex $w$-plane. We introduce and discuss this function in this section. Put

$$
\Omega:=\{w \in \mathbf{C}: \Im w<1\} \backslash\{i v: v \leq 0\},
$$

so that $\Omega=\Omega_{0}$ as defined in (2.3). We continue to use the convention that for $v<0$, $i v+$ denotes the point on the right side of the slit $\{i v: v<0\}$ and $i v$ - the point on the left side.

We define $h: \mathbf{C} \rightarrow \mathbf{R}$ first on $\mathbf{C} \backslash \Omega$ by

$$
h(w):=\left\{\begin{array}{cl}
|\Re w|-1 & \text { if } \Im w \geq 1, \\
0 & \text { if } \Re w=0 \text { and } \Im w \leq 0,
\end{array}\right.
$$

and then define $h$ on $\Omega$ as the solution of the Dirichlet problem for harmonic functions with the above boundary values. To ensure uniqueness, we also impose the growth condition

$$
h(w)=\mathcal{O}(|\Re w|) \quad(w \rightarrow \infty) .
$$

The mapping,

$$
z=\Phi(w):=\frac{i-w}{\sqrt{w^{2}-2 i w}},
$$

maps $\Omega$ conformally onto the upper half plane $\mathbf{H}=\{\Im z>0\}$. The line $w=u+i$, $u \in \mathbf{R}$ is mapped onto the interval $(-1,1)$. The function $h$ being harmonic in $\Omega$, yields by conformal transplantation the harmonic function $H$ on $\mathbf{H}$. The boundary values of $H$ are

$$
H(x)=\left\{\begin{array}{cl}
\frac{|x|}{\sqrt{1-x^{2}}}-1 & \text { if }-1<x<1, \\
0 & \text { elsewhere. }
\end{array}\right.
$$

Thus $H$ is given by the Poisson integral for the half plane

$$
H(x+i y)=\frac{1}{\pi} \int_{-1}^{1}\left(\frac{|s|}{\sqrt{1-s^{2}}}-1\right) \frac{y}{(x-s)^{2}+y^{2}} d s, \quad y>0 .
$$

To prove that $h$ is subharmonic on $\mathbf{C}$, we first need a lemma.

Lemma 7. For $v<0$,

$$
\frac{\partial h}{\partial u}(i v+)=\frac{2}{\pi} \arctan \left(\sqrt{v^{2}-2 v}\right)>0 .
$$

Proof. Using the map $\Phi$ from (3.3) and the chain rule, we get

$$
\frac{\partial h}{\partial u}(i v+)=\frac{1}{i} \Phi^{\prime}(i v+) \frac{\partial H}{\partial y}(\Phi(i v+)) .
$$

For $v<0$, it is easy to compute from (3.3)

$$
\Phi(i v+)=-\frac{1-v}{\sqrt{v^{2}-2 v}}, \quad \Phi^{\prime}(i v+)=\frac{i}{\left(v^{2}-2 v\right)^{3 / 2}} .
$$


Next we have from (3.4) for $|x|>1$,

$$
\begin{aligned}
\frac{\partial H}{\partial y}(x) & =\frac{1}{\pi} \int_{-1}^{1}\left(\frac{|s|}{\sqrt{1-s^{2}}}-1\right) \frac{1}{(x-s)^{2}} d s \\
& =\frac{1}{\pi} \int_{0}^{1} \frac{s}{\sqrt{1-s^{2}}}\left(\frac{1}{(x-s)^{2}}+\frac{1}{(x+s)^{2}}\right) d s-\frac{1}{\pi} \int_{-1}^{1} \frac{1}{(x-s)^{2}} d s \\
& =\frac{2}{\pi} \int_{0}^{1} \frac{s}{\sqrt{1-s^{2}}} \frac{x^{2}+s^{2}}{\left(x^{2}-s^{2}\right)^{2}} d s-\frac{2}{\pi} \frac{1}{x^{2}-1} \\
& =\frac{2}{\pi} \int_{0}^{1} \frac{x^{2}+1-t^{2}}{\left(x^{2}-1+t^{2}\right)^{2}} d t-\frac{2}{\pi} \frac{1}{x^{2}-1},
\end{aligned}
$$

where, for the last equality, we have made the change of variables $t=\sqrt{1-s^{2}}$. The integral of the rational function in (3.8) can be evaluated by elementary methods. The result is

$$
\frac{\partial H}{\partial y}(x)=\frac{2}{\pi} \frac{1}{\left(x^{2}-1\right)^{3 / 2}} \arctan \left(\frac{1}{\sqrt{x^{2}-1}}\right) .
$$

Combining this with (3.6) and (3.7), we obtain (3.5).

Proposition 8. The function $h$ is subharmonic on $\mathbf{C}$.

Proof. Since $h$ is continuous it is enough to show that $h$ satisfies locally the sub-meanvalue property:

$$
h\left(w_{0}\right) \leq \frac{1}{2 \pi} \int_{0}^{2 \pi} h\left(w_{0}+r e^{i \theta}\right) d \theta
$$

for every $w_{0} \in \mathbf{C}$ and $r$ sufficiently small (depending on $w_{0}$ ).

Since $h$ is harmonic in $\Omega$, the inequality (3.9) holds for $w_{0} \in \Omega$. Next, we note from (3.1) that $h(w) \geq|\Re w|-1$ for $w \notin \Omega$. Since $h$ is harmonic in $\Omega,|\Re w|-1$ is subharmonic, and (3.2) holds, we get

$$
|\Re w|-1 \leq h(w), \quad w \in \mathbf{C} .
$$

Hence, if $\Im w_{0} \geq 1$ and $r>0$,

$$
\begin{aligned}
h\left(w_{0}\right)=\left|\Re w_{0}\right|-1 & \leq \frac{1}{2 \pi} \int_{0}^{2 \pi}\left(\left|\Re\left(w_{0}+r e^{i \theta}\right)\right|-1\right) d \theta \\
& \leq \frac{1}{2 \pi} \int_{0}^{2 \pi} h\left(w_{0}+r e^{i \theta}\right) d \theta
\end{aligned}
$$

Thus (3.9) is satisfied if $\Im w_{0} \geq 1$.

Further, it follows from Lemma 7 and the symmetry of $h$ around the imaginary axis, that there is an open set $U$ containing the negative imaginary axis such that

$$
h(w) \geq 0, \quad w \in U .
$$

This clearly gives (3.9) for $w_{0}=i v, v<0$, if $r$ is small enough so that the circle with center $w_{0}$ and radius $r$ is contained in $U$.

Now it is proved that $h$ is subharmonic in $\mathbf{C} \backslash\{0\}$. However, since $h$ is continuous, this also implies subharmonicity at 0 , e.g., see [11, Theorem 3.6.1], and the proposition follows. 
For later use, we need an upper estimate on $h$. First we state a computational lemma.

Lemma 9. The function $v \mapsto h(i v), 0<v<1$, is decreasing.

Proof. The mapping $\Phi$ from (3.3) maps the segment $w=i v, 0<v<1$ onto the positive imaginary axis, and $v \mapsto h(i v)$ is decreasing for $v \in(0,1)$ if and only if $y \mapsto H(i y)$ increases for $y>0$.

We have from (3.4),

$$
H(i y)=\frac{2}{\pi} \int_{0}^{1} \frac{s}{\sqrt{1-s^{2}}} \frac{y}{s^{2}+y^{2}} d s-\frac{2}{\pi} \arctan \left(\frac{1}{y}\right) .
$$

The change of variables $t=\sqrt{1-s^{2}} / \sqrt{1+y^{2}}$ leads to

$$
\begin{aligned}
H(i y) & =\frac{2 y}{\pi \sqrt{1+y^{2}}} \int_{0}^{1 / \sqrt{1+y^{2}}} \frac{d t}{1-t^{2}}-\frac{2}{\pi} \arctan \left(\frac{1}{y}\right) \\
& =\frac{2 y}{\pi \sqrt{1+y^{2}}} \log \left(\frac{\sqrt{1+y^{2}}+1}{y}\right)-\frac{2}{\pi} \arctan \left(\frac{1}{y}\right) .
\end{aligned}
$$

Then, after some straightforward calculations,

$$
\frac{\partial H}{\partial y}(i y)=\frac{2}{\pi\left(1+y^{2}\right)^{3 / 2}} \log \left(\frac{\sqrt{1+y^{2}}+1}{y}\right),
$$

which is positive for $y>0$, and this proves the lemma.

Proposition 10. For every $w \in \mathbf{C}$, we have

$$
h(w) \leq|\Re w| .
$$

Proof. Because of (3.1), the inequality (3.10) is clear if $w \notin \Omega$. Since, by Lemma 8 , we have $h(i v) \leq h(0)=0$ for $0<v<1$, it follows that (3.10) also holds for $w=i v$, $0<v<1$. Setting

$$
\Omega_{+}:=\Omega \cap\{\Re w>0\} \quad \text { and } \Omega_{-}:=\Omega \cap\{\Re w<0\},
$$

we have (3.10) for $w \in \partial \Omega_{+}$. As both sides are harmonic in $\Omega_{+}$and we have the growth condition (3.2), the inequality (3.10) continues to hold throughout $\Omega_{+}$. Similarly, (3.10) holds for $w \in \Omega_{-}$, and the proposition follows.

\section{Proof of Theorem 1}

Proof of Theorem 1(a). It is enough to prove (2.1) for $\lambda=1$ since the general case follows from this special case by taking $f(x) / \lambda$. Let $\theta \in(-\pi / 2, \pi / 2)$. We consider the domain

$$
\Omega_{\theta}=\{w \in \mathbf{C}: \Im w<1\} \backslash\{i v: v \leq 1-\cos \theta\},
$$

which already appeared in (2.3), and the function

$$
h_{\theta}(w):=h\left(\frac{w-i(1-\cos \theta)}{\cos \theta}\right)
$$

where $h$ is the subharmonic function from Section 3. 
Let $f \in L^{1}$, and set

$$
F(z):=\frac{i}{\pi} \int_{-\infty}^{\infty} \frac{f(t)}{z-t} d t, \quad \Im z>0 .
$$

The analytic function $F$ belongs to the Hardy space $H_{1}$ of the upper half plane and has the a.e. non-tangential boundary values

$$
F(x)=f(x)+i \tilde{f}(x), \quad-\infty<x<\infty,
$$

e.g., see [8]. It follows from Proposition 8 and (4.1) that $h_{\theta}(F(z))$ is a subharmonic function in the upper half plane. It is easy to see that $h_{\theta}(F(z)) \rightarrow 0$ as $\Im z \rightarrow 0$; therefore,

$$
h_{\theta}(F(i y)) \leq \frac{1}{\pi} \int_{-\infty}^{\infty} h_{\theta}(F(x)) \frac{y}{x^{2}+y^{2}} d x, \quad y>0 .
$$

Since

$$
\lim _{y \rightarrow \infty} \pi y F(i y)=I(f),
$$

which is easy to verify from (4.2), we have

$$
\lim _{y \rightarrow \infty} \pi y h_{\theta}(F(i y))=I(f) \frac{\partial h_{\theta}}{\partial u}(0+)
$$

in case $I(f) \geq 0$, and

$$
\lim _{y \rightarrow \infty} \pi y h_{\theta}(F(i y))=I(f) \frac{\partial h_{\theta}}{\partial u}(0-)
$$

if $I(f) \leq 0$. Since $\partial h_{\theta} / \partial u(0+)=-\partial h_{\theta} / \partial u(0-)$, we obtain in all cases

$$
\lim _{y \rightarrow \infty} \pi y h_{\theta}(F(i y))=|I(f)| \frac{\partial h_{\theta}}{\partial u}(0+) .
$$

Then multiplying (4.3) by $\pi y$ and letting $y \rightarrow \infty$, and using (4.4) and the dominated convergence theorem, we get

$$
|I(f)| \frac{\partial h_{\theta}}{\partial u}(0+) \leq \int_{-\infty}^{\infty} h_{\theta}(F(x)) d x .
$$

Now let $E:=\{x \in \mathbf{R}: \tilde{f}(x) \geq 1\}$. Then for $x \in E$, we have $\Im F(x) \geq 1$, so that by (3.1) and (4.1),

$$
h_{\theta}(F(x))=\frac{1}{\cos \theta}|f(x)|-1, \quad x \in E .
$$

For $x \notin E$, we use (4.1) and Proposition 9 to obtain

$$
h_{\theta}(F(x)) \leq \frac{1}{\cos \theta}|f(x)|, \quad x \in \mathbf{R} \backslash E .
$$

Then combining (4.5)-(4.7), we get

$$
\begin{aligned}
|I(f)| \frac{\partial h_{\theta}}{\partial u}(0+) & \leq \int_{E} h_{\theta}(F(x)) d x+\int_{\mathbf{R} \backslash E} h_{\theta}(F(x)) d x \\
& \leq \int_{E}\left(\frac{1}{\cos \theta}|f(x)|-1\right) d x+\int_{\mathbf{R} \backslash E} \frac{1}{\cos \theta}|f(x)| d x \\
& =\frac{1}{\cos \theta}\|f\|_{1}-m(E) .
\end{aligned}
$$


To compute $\partial h_{\theta} / \partial u(0+)$, we use (4.1) and Lemma 7, which gives

$$
\begin{aligned}
\frac{\partial h_{\theta}}{\partial u}(0+) & =\frac{1}{\cos \theta} \frac{\partial h}{\partial u}\left(-i \frac{1-\cos \theta}{\cos \theta}+\right) \\
& =\frac{2}{\pi \cos \theta} \arctan \sqrt{\left(\frac{1-\cos \theta}{\cos \theta}\right)^{2}+2 \frac{1-\cos \theta}{\cos \theta}} \\
& =\frac{2|\theta|}{\pi \cos \theta} .
\end{aligned}
$$

Inserting this in (4.8) leads to

$$
m(E) \leq \frac{1}{\cos \theta}\|f\|_{1}-\frac{2|\theta|}{\pi \cos \theta}|I(f)|
$$

and this proves (2.1) with $\lambda=1$.

Proof of Theorem 1(b). Again it is enough to consider $\lambda=1$ only. For the function $f_{\theta}$ of $(2.2)$, it can be shown that

$$
\begin{aligned}
& \tilde{f}_{\theta}(x)=1, \quad \text { if }-1<x<1, \\
& \tilde{f}_{\theta}(x) \leq 1-\cos \theta, \quad \text { elsewhere. }
\end{aligned}
$$

Thus $m(\{x \in \mathbf{R}: \tilde{f}(x) \geq 1\})=2$. Using (2.11) and (2.12), it is easy to verify that equality holds in (2.1).

Alternatively, one can follow the proof of part (a) and verify that for any conformal mapping $F$ from $\mathbf{H}$ onto $\Omega_{\theta}$ satisfying (2.4) and for $f(x)=\Re F(x), x \in \mathbf{R}$, equality holds in all of the above inequalities. Thus equality holds in (2.1) for $f$. Since $f_{\theta}$ corresponds to such a conformal mapping, the theorem follows.

\section{Proofs of Theorem 5 and Corollary 6}

We start with two lemmas.

Lemma 11. Let $g, g_{n}, n=1,2, \ldots$ be real-valued measurable functions on $\mathbf{R}$ such that $g_{n}(x) \rightarrow g(x)$ for almost every $x \in \mathbf{R}$. Let $c>0$ and suppose that

$$
m\left(\left\{x \in \mathbf{R}: g_{n}(x) \geq \lambda\right\}\right) \leq \frac{c}{\lambda}, \quad \lambda>0, \quad n=1,2, \ldots .
$$

Then,

$$
m(\{x \in \mathbf{R}: g(x) \geq \lambda\}) \leq \frac{c}{\lambda}, \quad \lambda>0 .
$$

Proof. If $E_{\lambda}:=\{x \in \mathbf{R}: g(x)>\lambda\}$ and $E_{k, \lambda}:=\left\{x \in \mathbf{R}: \forall n \geq k g_{n}(x)>\lambda\right\}$, then $E_{k, \lambda} \subset E_{k+1, \lambda}, m\left(E_{k, \lambda}\right) \leq c / \lambda$ and $E_{\lambda} \backslash N \subset \cup_{k} E_{k, \lambda}$ where $N$ has zero Lebesgue measure. Thus $m\left(E_{\lambda}\right) \leq c / \lambda$. In the same way, we get $m\left(E_{\lambda-\epsilon}\right) \leq c /(\lambda-\epsilon)$ for every $\epsilon>0$. Letting $\epsilon \rightarrow 0$, we obtain the lemma.

Lemma 12. Let $n \in \mathbf{N}, \zeta_{1}, \ldots, \zeta_{n} \in \mathbf{C}, A_{1}, \ldots, A_{n} \in \mathbf{R}$ and $\sigma \in[-1,1]$ such that $\sum_{j=1}^{n} A_{j}=\sigma \sum_{j=1}^{n}\left|A_{j}\right|$. Then for

$$
g(x):=\sum_{j=1}^{n} A_{j} \Re\left(\frac{1}{x-\zeta_{j}}\right)
$$


and $\lambda>0$, we have

$$
m(\{x \in \mathbf{R}: g(x) \geq \lambda\}) \leq \frac{\pi K(\sigma)}{\lambda} \sum_{j=1}^{n}\left|A_{j}\right| .
$$

Proof. By virtue of Lemma 11, we may assume that the numbers $\zeta_{j}$ are all nonreal. We put $\zeta_{j}=a_{j}+i b_{j}$ with $b_{j} \neq 0$ and

$$
f(x):=\sum_{j=1}^{n} A_{j} \frac{\left|b_{j}\right|}{\left(x-a_{j}\right)^{2}+b_{j}^{2}}
$$

Then we have

$$
I(f)=\pi \sum_{j=1}^{n} A_{j}, \quad\|f\|_{1} \leq \pi \sum_{j=1}^{n}\left|A_{j}\right|,
$$

and $\tilde{f}=g$. Thus, by Theorem 1 ,

$$
m(\{x \in \mathbf{R}: g(x) \geq \lambda\}) \leq \frac{K(\tau)}{\lambda}\|f\|_{1} \leq \frac{\pi K(\tau)}{\lambda} \sum_{j=1}^{n}\left|A_{j}\right|
$$

where $\tau$ is such that $I(f)=\tau\|f\|_{1}$. From (5.3), it follows that $|\tau| \geq|\sigma|$, and since $K$ is an even function which decreases on $[0,1]$, this implies $K(\tau) \leq K(\sigma)$. Hence, (5.2) follows.

Proof of Theorem 5. Let $r_{n}=p_{n} / q_{n}$ be a rational function with $\operatorname{deg} p_{n} \leq \alpha n$ and $\operatorname{deg} q_{n} \leq \beta n$. We may assume that both $p_{n}$ and $q_{n}$ are real polynomials. Let $\xi_{j}$, $j=1, \ldots, \operatorname{deg} p_{n}$, denote the zeros of $p_{n}$ counted according to their multiplicities, and $\eta_{j}, j=1, \ldots, \operatorname{deg} q_{n}$, the zeros of $q_{n}$. Then

$$
\frac{r_{n}^{\prime}(x)}{r_{n}(x)}=\sum_{j=1}^{\operatorname{deg} p_{n}} \frac{1}{x-\xi_{j}}-\sum_{j=1}^{\operatorname{deg} q_{n}} \frac{1}{x-\eta_{j}}=\sum_{j=1}^{\operatorname{deg} p_{n}} \Re\left(\frac{1}{x-\xi_{j}}\right)-\sum_{j=1}^{\operatorname{deg} q_{n}} \Re\left(\frac{1}{x-\eta_{j}}\right) .
$$

If for $k \in \mathbf{N}$ we define,

$$
\begin{aligned}
g_{k}(x): & =\left(\alpha n-\operatorname{deg} p_{n}\right) \Re\left(\frac{1}{x-i k}\right)+\sum_{j=1}^{\operatorname{deg} p_{n}} \Re\left(\frac{1}{x-\xi_{j}}\right) \\
& -\left(\beta n-\operatorname{deg} q_{n}\right) \Re\left(\frac{1}{x-i k}\right)-\sum_{j=1}^{\operatorname{deg} q_{n}} \Re\left(\frac{1}{x-\eta_{j}}\right),
\end{aligned}
$$

then $g_{k}(x) \rightarrow r_{n}^{\prime}(x) / r_{n}(x)$ as $k \rightarrow \infty$. From Lemma 12 , we get for every $k$,

$$
m\left(\left\{x \in \mathbf{R}: g_{k}(x) \geq \lambda\right\}\right) \leq \frac{(\alpha+\beta) \pi}{\lambda} K\left(\frac{\alpha-\beta}{\alpha+\beta}\right) n .
$$

Then (2.13) follows from this and Lemma 11.

To prove that $(\alpha+\beta) \pi K((\alpha-\beta) /(\alpha+\beta))$ cannot be replaced by a smaller constant, we put $\sigma:=(\alpha-\beta) /(\alpha+\beta), \theta:=\theta_{\sigma}$, and we recall the function $f_{\theta}$ from (2.2):

$$
f_{\theta}(x)=\frac{x+\sin \theta}{\sqrt{1-x^{2}}}, \quad-1<x<1,
$$


and $f_{\theta}(x)=0$ elsewhere. Then $\tilde{f}_{\theta}(x)=1$ for $x \in(-1,1)$, while $\tilde{f}_{\theta}(x) \leq 1-\cos \theta$ for $|x|>1$. Hence,

$$
m\left(\left\{x \in \mathbf{R}: \tilde{f}_{\theta} \geq 1\right\}\right)=m\left(\left\{x \in[-1,1]: \tilde{f}_{\theta} \geq 1\right\}\right)=2 .
$$

Associated with $f_{\theta}$ is the conformal map $F$ from $\mathbf{H}$ onto $\Omega_{\theta}$ such that

$$
F(x)=f_{\theta}(x)+i \tilde{f}_{\theta}(x), \quad-\infty<x<\infty .
$$

We note that for all $x$ except \pm 1 ,

$$
\lim _{\delta \rightarrow 0} \Im F(x+i \delta)=\tilde{f}_{\theta}(x) .
$$

Hence, by (5.5), we can find for any given $\epsilon>0$, a $\delta>0$ such that

$$
m(\{x \in[-1,1]: \Im F(x+i \delta)>1-\epsilon\}) \geq 2-\epsilon .
$$

The construction of suitable rational functions $r_{n}$ will result from discretizing the signed measure $f_{\theta}(t) d t$ which is supported on $[-1,1]$. We note that its positive part is on $[-\sin \theta, 1]$ with total positive mass

$$
\int_{-\sin \theta}^{1} f_{\theta}(t) d t=\frac{1+\sigma}{K(\sigma)}
$$

The negative part is on $[-1,-\sin \theta]$ and

$$
\int_{-1}^{-\sin \theta} f_{\theta}(t) d t=-\frac{1-\sigma}{K(\sigma)}
$$

This may be obtained from either (2.8), (2.11), and (2.12) or by direct calculation. For a given $n$, we choose $[\alpha n / 2]$ points $x_{1, n}, \ldots, x_{[\alpha n / 2], n}$ in $(-\sin \theta, 1]$ satisfying

$$
x_{1, n}=1 \quad \text { and } \quad \int_{x_{j+1, n}}^{x_{j, n}} f_{\theta}(t) d t=\frac{1}{[\alpha n / 2]} \frac{1+\sigma}{K(\sigma)}, \quad j=1, \ldots,[\alpha n / 2]-1 .
$$

Similarly, we choose $[\beta n / 2]$ points $y_{1, n}, \ldots, y_{[\beta n / 2], n}$ in $[-1,-\sin \theta)$ according to

$$
y_{1, n}=-1 \quad \text { and } \quad \int_{y_{j, n}}^{y_{j+1, n}} f_{\theta}(t) d t=-\frac{1}{[\beta n / 2]} \frac{1-\sigma}{K(\sigma)}, \quad j=1, \ldots,[\beta n / 2]-1 \text {. }
$$

These points are well-defined because of (5.7) and (5.8). Let $\nu_{n}$ be the signed measure on $[-1,1]$ with mass $2 / n$ at each $x_{j, n}$ and mass $-2 / n$ at each $y_{j, n}$. Using (5.7)-(5.10), we find that

$$
\lim _{n \rightarrow \infty} \nu_{n}=\frac{\alpha+\beta}{2} K(\sigma) f_{\theta}(t) d t
$$

where the convergence is in the sense of weak* convergence of signed measures on $[-1,1]$.

Now, for every $n$, we introduce the rational function

$$
r_{n}(x):=\frac{\prod_{j=1}^{[\alpha n / 2]}\left[\left(x-x_{j, n}\right)^{2}+\delta^{2}\right]}{\prod_{j=1}^{[\beta n / 2]}\left[\left(x-y_{j, n}\right)^{2}+\delta^{2}\right]}
$$


Thus, the degree of the numerator is $\leq \alpha n$ and the degree of the denominator is $\leq \beta n$. By the definition of the measure $\nu_{n}$,

$$
\frac{r_{n}^{\prime}(x)}{n r_{n}(x)}=\int \frac{x-t}{(x-t)^{2}+\delta^{2}} d \nu_{n}(t), \quad-\infty<x<\infty .
$$

As $n \rightarrow \infty$, we find from (5.11) for every $x$,

$$
\begin{aligned}
\lim _{n \rightarrow \infty} \frac{r_{n}^{\prime}(x)}{n r_{n}(x)} & =\frac{\alpha+\beta}{2} K(\sigma) \int \frac{x-t}{(x-t)^{2}+\delta^{2}} f_{\theta}(t) d t \\
& =\frac{\alpha+\beta}{2} \pi K(\sigma) \Im F(x+i \delta) .
\end{aligned}
$$

Then it follows from (5.6) that when $n$ is sufficiently large,

$$
m\left(\left\{x \in[-1,1]: \frac{2 r_{n}^{\prime}(x)}{(\alpha+\beta) \pi K(\sigma) n r_{n}(x)}>1-\epsilon\right\}\right) \geq 2-\epsilon .
$$

That is, with $\lambda:=((\alpha+\beta) / 2) \pi K(\sigma)(1-\epsilon) n$,

$$
m\left(\left\{x \in[-1,1]: \frac{r_{n}^{\prime}(x)}{r_{n}(x)}>\lambda\right\}\right) \geq \frac{(2-\epsilon)(1-\epsilon)(\alpha+\beta) \pi K(\sigma) n}{2 \lambda} .
$$

This shows that $(\alpha+\beta) \pi K(\sigma)$ cannot be replaced by a constant less than $(2-\epsilon)(1-$ $\epsilon)(\alpha+\beta) \pi K(\sigma) / 2$. Since $\epsilon>0$ can be taken arbitrarily small, the theorem follows.

Proof of Corollary 6. To obtain a contradiction, we let

$$
R>(\alpha+\beta) \pi K\left(\frac{\alpha-\beta}{\alpha+\beta}\right),
$$

and we suppose there exist $r_{n}=p_{n} / q_{n}$ with $\operatorname{deg} p_{n} \leq \alpha n$ and $\operatorname{deg} q_{n} \leq \beta n$ such that (2.14) holds. Then $r_{n}(x)>0$ on $[0, R]$ for $n$ large enough, and taking logarithms, we have

$$
\lim _{n \rightarrow \infty}\left\|\log r_{n}(x)-n x\right\|_{[0, R]}=0 .
$$

By (5.13), we can choose $\epsilon, \delta>0$ such that

$$
\frac{(\alpha+\beta) \pi}{1-\delta} K\left(\frac{\alpha-\beta}{\alpha+\beta}\right)<R-\epsilon \text {. }
$$

Then from (2.13) with $\lambda=(1-\delta) n$, we get

so

$$
m\left(\left\{x \in \mathbf{R}: \frac{r_{n}^{\prime}(x)}{r_{n}(x)} \geq(1-\delta) n\right\}\right)<R-\epsilon,
$$

$$
m\left(\left\{x \in[0, R]: \frac{r_{n}^{\prime}(x)}{r_{n}(x)}<(1-\delta) n\right\}\right) \geq \epsilon .
$$

The set where $r_{n}^{\prime} / r_{n}<(1-\delta) n$ is the union of $\mathcal{O}(n)$ intervals. Thus, it follows from (5.15) that there exist $a_{n}, b_{n} \in[0, R]$ and $\rho>0$ such that

and $b_{n}-a_{n}>\rho / n$. Then

$$
\frac{r_{n}^{\prime}(x)}{r_{n}(x)}<(1-\delta) n, \quad \text { for } x \in\left(a_{n}, b_{n}\right)
$$

$$
\log r_{n}\left(b_{n}\right)-\log r_{n}\left(a_{n}\right)=\int_{a_{n}}^{b_{n}} \frac{r_{n}^{\prime}(x)}{r_{n}(x)} d x<(1-\delta) n\left(b_{n}-a_{n}\right)
$$


therefore,

$$
\left(\log r_{n}\left(b_{n}\right)-n b_{n}\right)-\left(\log r_{n}\left(a_{n}\right)-n a_{n}\right)<-\delta n\left(b_{n}-a_{n}\right)<-\delta \rho<0 .
$$

Then (5.14) cannot hold, and the corollary follows from this contradiction.

Acknowledgements. The author was supported by a postdoctoral fellowship of the Belgian National Fund for Scientific Research, Scientific Research Network nr WO.011.96N: Fundamental Methods and Techniques in Mathematics.

\section{References}

1. A. Baernstein II, Some sharp inequalities for conjugate functions, Indiana Univ. Math. J. 27 (1978), 833-852.

2. P. B. Borwein, The size of $\left\{x: r_{n}^{\prime} / r_{n} \geq 1\right\}$ and lower bounds for $\left\|e^{-x}-r_{n}\right\|$, J. Approx. Theory 36 (1982), 73-80.

3. P. B. Borwein and T. Erdélyi, Polynomials and Polynomial Inequalities, Springer-Verlag, Berlin, 1995.

4. P. B. Borwein, E. A. Rakhmanov, and E. B. Saff, Rational approximation with varying weights I, Constr. Approx. 12 (1996), 223-240.

5. B. Davis, On the distribution of conjugate functions of nonnegative measures, Duke Math. J. 40 (1973), 695-700.

6. (1974), 307-311.

7. A. A. Gonchar and E. A. Rakhmanov, Equilibrium distributions and degree of rational approximation of analytic functions, Math. USSR Sbornik 62 (1989), 305-348.

8. P. Koosis, Introduction to $H_{p}$ Spaces, Cambridge University Press, Cambridge, 1980.

9. G. K. Kristiansen, Solution to problem 80-16, SIAM Review 24 (1982), 77-78.

10. E. A. Rakhmanov, E. B. Saff, and P. C. Simeonov, Rational approximation with varying weights II, J. Approx. Theory 92 (1998), 331-338.

11. T. Ransford, Potential Theory in the Complex Plane, Cambridge University Press, Cambridge, 1995.

Departement Wiskunde, Katholieke Universiteit Leuven, Celestijnenlaan 200 B, B-3001 LeUven (HeVerleE), Belgium 